\title{
Spatial contiguity facilitates Pavlovian conditioning
}

\author{
JOHN CHRISTIE \\ Dalhousie University, Halifax, Nova Scotia, Canada
}

\begin{abstract}
The spatial contiguity between Pavlovian conditioned and unconditioned stimuli was examined using a chamber designed to prevent the usual confounding with delay of reinforcement. Eight ring doves were autoshaped in this apparatus. For half of the trials, a conditioned stimulus (CS) was presented $11 \mathrm{~cm}$ from the unconditioned stimulus (US); for the other trials, another CS was presented $74 \mathrm{~cm}$ away. After extended training, the stimuli were presented in nonreinforced test trials at a common, intermediate $(42 \mathrm{~cm})$ position. The CS that had been nearer the US was approached more closely, indicating that higher spatial contiguity facilitates Pavlovian conditioning.
\end{abstract}

In studies of Pavlovian conditioning, one factor that has yet to be successfully isolated and examined is the spatial contiguity between a conditioned stimulus (CS) and an unconditioned stimulus (US). Ever since earliest associationist theories, spatial contiguity has been assumed to be a relevant factor in conditioning, but little direct empirical evidence has been acquired. A review by Mackintosh (1983) described it as an established fact that a near CS produces better conditioning than a distant one, but in his review Mackintosh cited only one direct study (Rescorla \& Cunningham, 1979, examined below). Principally, Mackintosh supported his argument by referring to various experiments where spatial contiguity $a p$ peared to be an important factor.

The main difficulty in testing spatial contiguity in Pavlovian conditioning is that when a CS is spatially distant from a US, any animal traveling from the former to the latter experiences a temporal delay of reward, a manipulation that is known to impede conditioning. ${ }^{1}$ Therefore, when spatial contiguity has been manipulated, temporal contiguity has always been confounded. Because of this confound, it remains possible that the important factor in conditioning is the time between encounters of the CS and US. Spatial contiguity, therefore, could play no role, because it is highly unlikely that a natural situation could arise where it is not directly related to temporal contiguity.

Perhaps it is because of the difficulty of overcoming the confounding of temporal and spatial contiguity that experiments manipulating the latter factor have been reported only once, and both of those experiments involved a

This research was funded by a grant from the National Science and Engineering Research Council to B. R. Moore, who also assisted in designing the apparatus. Thanks are extended to Tracy Taylor, Billy Schmidt, and B. R. Moore for suffering through drafts of this manuscript and providing helpful insights to improve the writing. Thanks are also extended to Bob Barnet for help in clarifying this paper and inspiring some of the ideas for future research. Correspondence should be sent to the author at the Department of Psychology, Dalhousie University, Halifax, NS, Canada B3H 4J1 (e-mail: jc@or.psychology.dal.ca). second-order conditioning paradigm. In Experiment 1 of Rescorla and Cunningham (1979), pigeons were shaped to a colored light (CS1), and that CS was subsequently used as a reinforcer to condition responding to either of two second-order stimuli (CS2s). Two stimulus protectors were used for the second phase of the experiment, each of which could present a light of any of three colors (two CS2s and one CS1). The subjects were presented with a CS2 followed by a CS1 at the same site or elsewhere. It was found that second-order conditioning was faster with spatial contiguity (CS2 and CS1 in same location) even though the terminal response rates were the same. This was taken as evidence that spatial contiguity facilitates secondorder Pavlovian conditioning.

There are, however, two problems with this conclusion. It remains possible that subjects approached and attempted to peck the first-order CS in Rescorla and Cunningham's (1979) Experiment 1, and that in the spatially discontiguous condition pecking the second-order stimulus delayed performance of this behavior. This temporal delay may have affected conditioning. Furthermore, response competition (perhaps arising from anticipatory movements toward CS1) may have reduced pecking toward the CS2 in the discontiguous condition. In short, reduced pecking may have occurred to both CS1 and CS2 in the discontiguous condition for reasons other than lack of spatial contiguity.

In an attempt to address the problems of their Experiment 1, Rescorla and Cunningham (1979) performed a second experiment in which they used Pavlovian conditioned suppression. As in Experiment 1, this was a secondorder conditioning paradigm, and it was performed in the same apparatus. In Stage 1, subjects were conditioned to suppress pecking at a light above a food site, with shock to the leg as the US. In Stage 2, the CS1 was paired with a CS2 at either a spatially contiguous or spatially discontiguous site. It was found that there was greater suppression with the CS2 that occurred in the same location as the CS1 than there was with the discontiguous CS2.

This design did not correct the problems of Experiment 1 . As with Experiment 1, part of the effects observed 
may have been due to response competition by anticipatory behavior, but this time it was in the spatially contiguous condition. The subjects may have been anticipating the spatially contiguous $\mathrm{CS} 1$ and reduced pecking accordingly. This type of behavior is impossible to distinguish from conditioning in their paradigm. Furthermore, the temporal confounding still remains. In the case of Experiment 1, it takes the subject longer to get to the far CS1 than to the near, a confounding that may reduce the amount of conditioning observed in the discontiguous condition. In Experiment 2, subjects will be able to immediately avoid the CS1 in the spatially discontiguous condition because it appears at a different location, but will be slowed in their ability to avoid CS1 in the near condition because it appears at their present location. Encountering CS1 is necessary for conditioning; therefore, there should be stronger suppression in the spatially contiguous condition due to temporal contiguity.

A solution to the problem of response competition in both of Rescorla and Cunningham's (1979) experiments would have been to select specific sites for the CS1 and CS2 during the training phase rather than varying them within subject. Nonreinforced test trials could then be given at an intermediate site exactly halfway between these two training sites (it would be farther away for a contiguous CS and nearer for a discontiguous CS). In that situation the amount of conditioning could be measured with response competition controlled. Recognizing the potential of response competition to taint the outcome of spatial contiguity manipulations, a testing procedure similar to that described above was used in the experiment to be reported here.

In the present experiment, the spatial distance between a CS and US was manipulated without confounding delay of reinforcement. The problem was addressed by using a specially designed conditioning chamber. The principle underlying the apparatus design was that the distance traveled between any two points placed on the circumference of a circle is always equal if that distance is measured through an intermediate point, the center. The subject's CSs and USs were all presented on the circumference of a circle, and the subject was required to travel through the center of that circle to collect reinforcers. Thus, the time between conditioned response (CR) and unconditioned response (UR) (and thus the US) was held constant while the contiguity of the CS and US was varied along the circumference of the circle. ${ }^{2}$

\section{METHOD}

\section{Subjects}

Eight ring doves (Streptopelia risoria) of unknown sex served as subjects. They were maintained at $85 \%$ of their free-feeding weight, individually housed, and maintained on a 12/12 light/dark cycle.

\section{Apparatus}

All stimulus presentation and timing was controlled by electromagnetic equipment in a separate control room some distance away.

A sketch of an overhead view of the conditioning chamber is shown in Figure 1. All exterior walls and the floor were made of unfinished plywood, except for the curved perimeter wall, which was constructed of two strips of white Plexiglas. The lower strip of Plexiglas on the curved wall extended $11 \mathrm{~cm}$ from the floor, and the higher strip protruded $2 \mathrm{~cm}$ from the ceiling. With a $3-\mathrm{cm}$ space between the Plexiglas strips to access CSs, this sums to an interior height of $16 \mathrm{~cm}$. In the top, which was made of $1 / 2$-in. hardware cloth, there were two doors. One door, $16 \mathrm{~cm}$ square, was above the starting platform, for placement and removal of the subject; another door $(9 \times 15 \mathrm{~cm})$ was over the food site, with the long axis perpendicular to that of the food alley.

The CSs were projected onto LeHigh Valley response keys by I.D.O. stimulus projectors. The projectors were centered $12 \mathrm{~cm}$ from the floor, and mounted in $12-\mathrm{cm}$-wide matte black aluminum stands. The red or white CS could be viewed and pecked through a $3-\mathrm{cm}$ hole in the stand. The straight-line distances from the food cup center to each of the CS location centers were 11,42 , and $74 \mathrm{~cm}$ for the near, middle, and far locations, respectively.

The 16-cm-high wall dividing the food alley and the CS sites was made of plywood for Doves 1-4 (Apparatus 1). For Doves 5-8, a 0.5 in. hardware cloth partition was used (Apparatus 2.). The starting platform and a removable (larger) pretraining platform were constructed of sheet aluminum and raised $3 \mathrm{~mm}$ from the floor. The platform rested on microswitches that controlled a 3-sec timer. The sheet aluminum food cup cover, which concealed a $2-\mathrm{cm}$-square $\times 3$-mm-deep hole containing $0.5 \mathrm{~g}$ of red and white millet, was a spring-loaded slide that could be released by a solenoid.

\section{Procedure}

The subjects were first hand-shaped during pretraining sessions to stay on the large pretraining platform for 3 consecutive seconds in order to receive the food. Once they accomplished that task, they were shaped to start on the platform shown in Figure 1.

During subsequent conditioning sessions, there were two possible $\mathrm{CSs}$, one at the near location and one at the far location (see Figure 1). For a given bird, one stimulus was always red and one was always white (counterbalanced across birds). After the subject had stood on the starting platform for the 3 consecutive seconds, one of the two CSs was presented for $5 \mathrm{sec}$ and followed by the US presentation. The US could be collected by the animal at any time after presentation; thus, late arrival at the reinforcer did not influence the quantity of reinforcement.

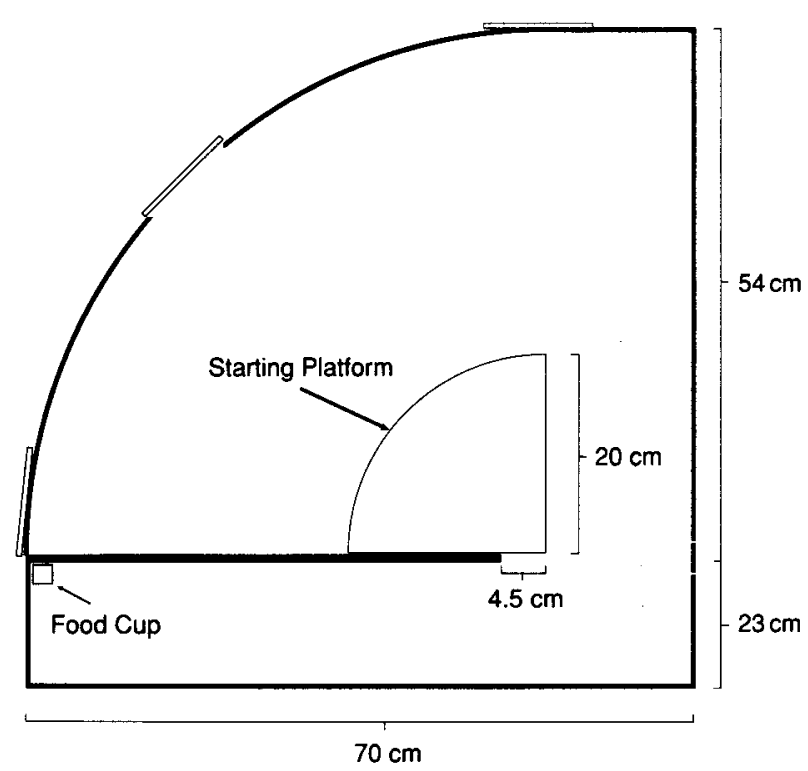

Figure 1. An overhead view of the apparatus used in this experiment. The heavy line drawn horizontally between the food cup and the platform represents a wall that was made of plywood for 4 of the subjects and 0.5 -in. hardware cloth for the other 4 subjects. The height of the apparatus was equal to the height of the CS panel. 
Table 1

Summary of Approaches to CS During Test Days

\begin{tabular}{crrcccc}
\hline \multirow{2}{*}{$\begin{array}{c}\text { Dove } \\
\text { Number }\end{array}$} & \multicolumn{2}{c}{ Mean Proximity } & & \multicolumn{2}{c}{ No. of Approaches } & \\
\cline { 2 - 3 } & Near CS & Far CS & & Near CS & Far CS & Partition \\
\hline 1 & 7.1 & 9.1 & 10 & 7 & plywood \\
2 & 8.4 & 10.9 & 10 & 8 & plywood \\
3 & 10.2 & 16.8 & 10 & 5 & plywood \\
4 & 11.9 & 8.3 & 10 & 7 & plywood \\
$M$ & 9.4 & 11.3 & 10 & 6.8 & \\
5 & 5.9 & 8.8 & 10 & 8 & wire mesh \\
6 & 10.8 & 8.5 & 6 & 6 & wire mesh \\
7 & 11.2 & 14.3 & 10 & 3 & wire mesh \\
8 & 4.2 & 12.4 & 10 & 10 & wire mesh \\
$M$ & 8.0 & 11.0 & 9 & 6.8 & \\
Pooled $M$ & 8.7 & 11.1 & 9.5 & 6.8 & \\
\hline
\end{tabular}

Note-The proximity to the CS is given in centimeters and the number of approaches is out of a possible 10 .

Each session contained 20 trials, 10 with each CS. Sequences of near and far CSs were randomized separately for each bird. Testing began after 30 sessions for the birds in Apparatus 1 and after 15 sessions for those trained in Apparatus 2. ${ }^{3}$ Test days were the same as nontest days except that the CS lights were presented at the location midway between the original positions and no reinforcers were delivered. There were lines on the floor of the cage at distances of $2,4,6, \ldots 24 \mathrm{~cm}$ from the CS. On every trial that the animal approached the CS, the proximity to the CS was visually scored by the experimenter. Trials were also rescored from videotape by an observer blind to the experimental conditions (the observer could not see the CS from the camera angle used)

\section{RESULTS}

There was on average a \pm 0.5 -cm difference between the experimenter's measurements and those of the independent, blind observer.

Doves often approached but did not contact the CSs, so approach frequency and proximity were used as the measure of conditioning. Table 1 summarizes the mean proximity of CS approach, and the number of approaches made during testing. The proximity of approach to the near CS color was marginally closer than that to the far CS $(8.7$ vs. $11.1 \mathrm{~cm})[t(7)=1.74, p=.06]$, and the near CS color was approached more often than the far CS color $(95 \%$ vs. $68 \%)[t(7)=3.27, p<.01]$.

\section{DISCUSSION}

During the test session, when CSs were equidistant from the US, subjects tended to more closely and more consistently approach the CS that had been near the food site than they did the CS that had been farther away. Thus, the degree of conditioning was stronger for the near CS than for the far CS, indicating that spatial contiguity facilitates Pavlovian conditioning.

The near CS and US were as close together as in normal conditioning chambers for pigeons. Lack of pecking in the near condition indicates that travel time or distance is also important.

Exactly what aspects of spatial contiguity are important remains to be discovered. Is absolute distance the critical factor in spatial contigu- ity or is it the visual angle relative to the animal? Would a depth difference provide the same results? Is the effect species specific? How would an animal with different cognitive mapping capabilities (i.e., landmarking) perform in a similar experiment? These are a few examples of the many important questions about spatial contiguity that require further inquiry.

Alternative methods one might implement in further explorations could use spatial contiguity as a variable in a design that used a simple barrier to make CS approach impossible (Kirby, 1968, cited in Moore, 1973). In this situation, spatial contiguity could be manipulated without creating a temporal confound, because a conditioned approach response is prevented. The response to the CSs at some midlocation could be measured after the barrier was removed or, alternatively, the CSs could then be used as a first-order stimulus in a second-order conditioning paradigm. If a CS that had been previously conditioned with close contiguity elicits better conditioning to a second-order stimulus than one conditioned farther from the US, then these results could provide additional evidence for the effects of spatial contiguity (Barnet, Arnold, \& Miller, 1991). Such a method might be more convenient than that employed here. Additionally, spatial contiguity (and many other factors in Pavlovian conditioning) might be more easily controlled in a paradigm in which the animal was restrained and biomedical CRs were measured, as in Pavlov's early experiments with dogs. These methods, however, are not generally as humane as autoshaping or as inexpensive.

It would be of interest to test this effect in species other than doves. It may be unimportant to some species that a CS is near or far from the US, or some species might simply map space in such a way that time to reach a location in space was more important than contiguity per se. The current experiment offers a beginning at judging whether spatial factors matter when all other things are held equal.

\section{REFERENCES}

Barnet, R. C., Arnold, H. M., \& Miller, R. R. (1991). Simultaneous conditioning demonstrated in second-order conditioning: Evidence for similar associative structure in forward and simultaneous conditioning. Learning \& Motivation, 22, 253-268.

Mackintosh, N. J. (1983). Conditioning and associative learning. Oxford: Oxford University Press, Clarendon Press.

MOORE, B. R. (1973). The role of directed Pavlovian reactions in simple instrumental learning in the pigeon. In R. A. Hinde \& J. S. Hinde (Eds.), Constraints on learning: Limitations and predispositions (pp. 159-188). New York: Academic Press.

Rescorla, R. A., \& Cunningham, C. L. (1979). Spatial contiguity facilitates second-order Pavlovian conditioning. Journal of Experimental Psychology: Animal Behaviowr Processes, 5, 152-161.

\section{NOTES}

1. An extensive discussion of all the difficulties with studies that have examined spatial contiguity or are thought to reveal something about it would not be appropriate in this brief report; instead, it will be focused primarily on research that most resembles the present design.

2. The interval between UR and CR would vary somewhat depending on the CR. If the CR toward a given CS becomes stronger, then the US-UR will be delayed and temporal contiguity will be reduced. Thus, the method requires that a spatial contiguity effect be powerful enough to overcome a loss of temporal contiguity.

3. The large difference in number of sessions is because the birds in the apparatus version with a plywood dividing wall were run first. I was hoping that the apparent ceiling behavior seen at around Session 15 in that apparatus was merely a plateau and continued running the birds for a prolonged period in pursuit of observing pecking behavior.

(Manuscript received July 10, 1995; revision accepted for publication March 5, 1996.) 